\title{
Total infrared luminosity estimation from local galaxies in AKARI all sky survey (Corrigendum)
}

\author{
A. Solarz ${ }^{1}$, T. T. Takeuchi ${ }^{2}$, and A. Pollo ${ }^{1,3}$ \\ 1 National Center for Nuclear Research, ul. Hoża 69, 00-681 Warsaw, Poland \\ e-mail: aleksandra.solarz@ncbj.gov.pl \\ 2 Division of Particle and Astrophysical Science, Nagoya University, Furo-cho, Chikusa-ku, 464-8602 Nagoya, Japan \\ 3 The Astronomical Observatory of the Jagiellonian University, ul. Orla 171, 30-244 Kraków, Poland
}

A\&A 592, A155 (2016), DOI: 10.1051/0004-6361/201629062

Key words. infrared: galaxies - galaxies: star formation - galaxies: evolution - errata, addenda

We correct an error present in the entry of the expressions for recalibration of the formulae to derive the total infrared luminosities using two and three AKARI FIS filters (Eqs. (6) and (7)). While the calculations and coeficients are correct, the relations reported in the original paper were expressed for the wrong (opposite) number of filters.

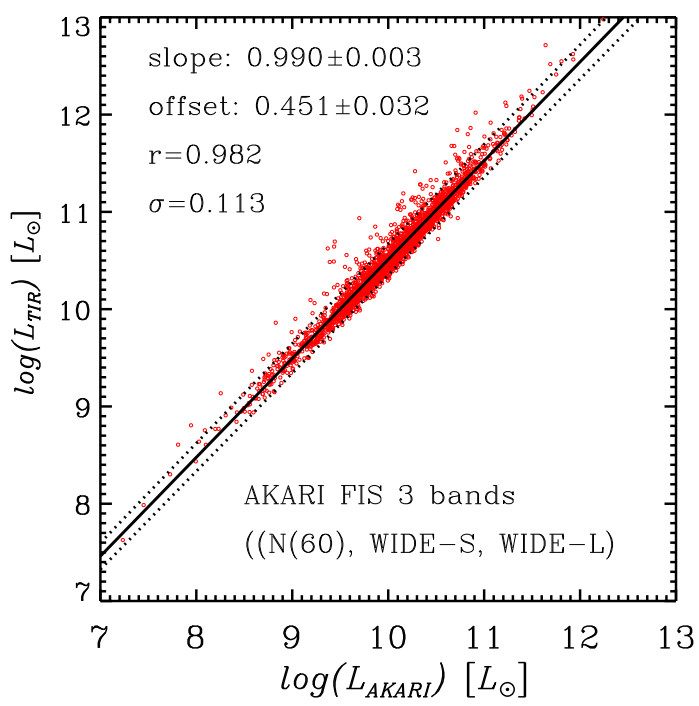

Fig. 1. Figure 3 should be replaced with notation presented here.
The proper relations which should replace Eqs. (6) and (7) are as follows:

$\log \left(L_{\mathrm{TIR}}\right)=(1.016 \pm 0.004) \log \left(L_{\mathrm{AKARI}}^{2 \mathrm{bands}}\right)+(0.349 \pm 0.040)$,

and

$\log \left(L_{\mathrm{TIR}}\right)=(0.990 \pm 0.003) \log \left(L_{\mathrm{AKARI}}^{\text {3bands }}\right)+(0.451 \pm 0.032)$.

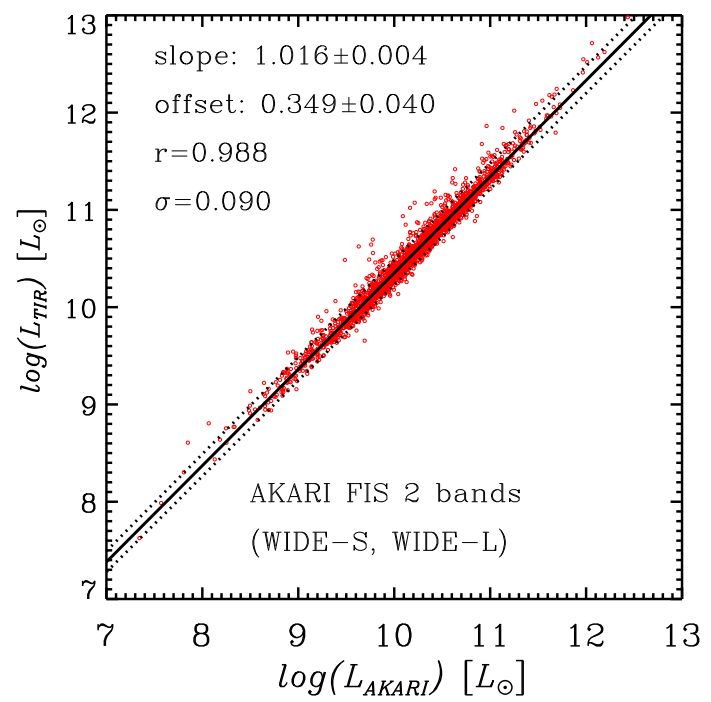

